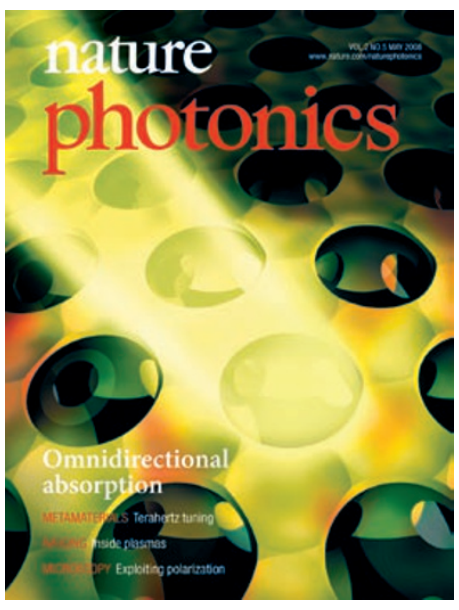

\section{Cover story}

Vol.2 No.5 May 2008

The ability to strongly absorb light over a wide range of angles of incidence and for any polarization is important for applications ranging from increasing the efficiency of solar cells through to optimizing thermal-emission sources and shielding in microphotonic circuits. Now Tatiana Teperik and co-workers have demonstrated a surface that can do just that. The surface is made from a layer of nanostructured gold, which contains an array of tiny spherical voids around $500 \mathrm{~nm}$ in diameter. It provides omnidirectional absorption by converting incident light into localized plasmons, which beome trapped and then dissipate. The particular wavelength that is absorbed is controlled by the thickness of the gold covering the voids. [Letter p299]

\section{IMAGING PLASMAS}

Laser-created plasmas are useful tools for generating beams of electrons, ions, $\mathrm{X}$-rays or attosecond pulses, but experimentally investigating the internal dynamics of such plasmas is a challenge. In this issue, Martin Centurion and co-workers report a technique for real-time imaging of the electric-field distribution of plasmas ionized by optical fields. The method relies on illuminating the plasma with a beam of electrons and monitoring the deflection of their trajectories caused by the plasma's electric-field distribution. With a temporal resolution of just $2.7 \mathrm{ps}$, the technique enables the capture of ultrafast events and their evolution. For example, Centurion et al. observe dips and lobes in the field distribution that cannot be easily observed by other means.

[Letter p315; News \& Views p272]

\section{TUNING ANTENNAS}

In the world of radiofrequency electronics, an antenna's resonance is often tuned to a desired frequency by using lumped circuit elements, such as capacitors and inductors, to alter the impedance. It now appears that a similar approach can be applied to optical nanoantennas in the world of photonics, by integrating tiny nanodisks and nanocylinders made from gold, silicon or silica into the antenna. In this issue, Andrea Alù and Nader Engheta demonstrate with theoretical simulations how the use of such nanocircuit elements can alter the frequency response of an optical antenna. The results suggest that optical engineers may soon be able to translate many ideas from analog electronic-circuit design into the optical realm. The result could be a breed of miniaturized optical circuits that can offer new functionalities or outperform existing optical designs.

[Letter p307; News \& Views p270]

\section{SUPERCONDUCTOR COUNTING}

Being able to count the exact number of photons in a low light flux is important for applications within quantum information processing and communications, however it's tricky to do. Fortunately, the task may be about to get easier thanks to Aleksander Divochiy and co-workers, who have made a parallel nanowire detector that relies on several tiny superconducting nanowires to do the job. Photons striking the wires, which are operated close to their critical current, cause the generation of a voltage that is proportional to the number of photons. The detector can count up to four photons at telecommunications wavelengths with a repetition rate of $80 \mathrm{MHz}$ and a sensitivity of the order of $10^{-18} \mathrm{~W} \mathrm{~Hz}^{-1 / 2}$. Divochiy and his co-workers also believe that devices optimized for counting more photons could prove useful for bridging the gap between single-photon-counting equipment and traditional low-light detectors. The limitation is that the superconducting nature of the device

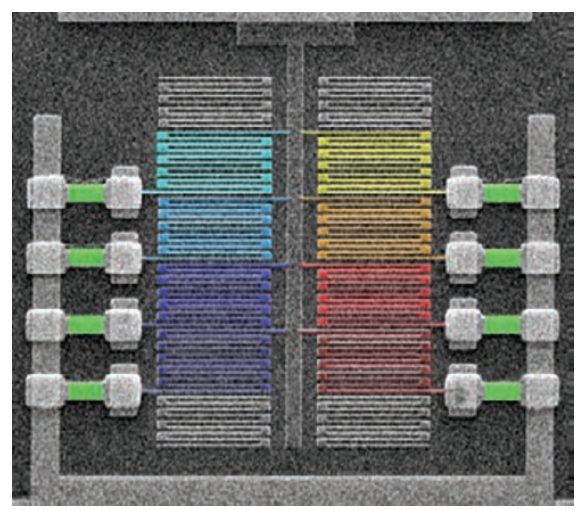

A scanning electron microscopy image of a parallel nanowire detector that can resolve the number of incident photons. means that it needs cooling to cryogenic temperatures of just a few Kelvin. [Letter p302; News \& Views p268]

\section{AGILE METAMATERIALS}

A metamaterial with a resonance in the terahertz regime that can be optically tuned by applying incident light could soon provide a way to create dynamic terahertz filters. The design by Hou-Tong Chen and co-workers takes the well-known split-ring element design of metamaterials and adds a twist - two tiny plates of silicon that create a capacitor within each element. When illuminated, photoexcited carriers in the silicon alter the effective capacitance of the split-ring element, thus tuning the resonant frequency of the metamaterial. Chen and co-workers report tuning from $850 \mathrm{GHz}$ to $1.06 \mathrm{THz}$, a range of $20 \%$. They also postulate that a similar effect, but reaching higher frequencies, can be achieved by using inductor elements.

[Letter p295; News \& Views p267; Interview p324]

\section{EXPLOITING POLARIZATION}

By carefully controlling the polarization of light to create an asymmetrically shaped focal spot, Derryck Reid and colleagues have shown that it is possible to image the features of a silicon integrated circuit with unprecedented detail. Their approach uses a high-numerical-aperture solid immersion lens to focus polarized light onto an integrated circuit to enable imaging of tiny buried transistors. It provides a resolution that exceeds the scalar diffraction limit, commonly known as Sparrow's criterion. Using an infrared laser operating at $1,530 \mathrm{~nm}$ the researchers show that there is a twofold difference in the resolution that can be obtained between light of orthogonal polarization states, and achieve an imaging resolution as high as around $120 \mathrm{~nm}$.

[Letter p311; News \& Views p273] 